\title{
Isópodos litorales y de aguas someras de la bahía de Todos los Santos, Baja California, México
}

\author{
Littoral and shallow water isopods from Todos los Santos Bay, Baja California, Mexico
}

\author{
Ernesto Campos $^{1 *}$ y Guillermo Villarreal ${ }^{2}$ \\ ${ }^{1}$ Facultad de Ciencias. Universidad Autónoma de Baja California, Apartado postal 296, Ensenada, Baja California, México. \\ ${ }^{2}$ Facultad de Ciencias Marinas. Universidad Autónoma de Baja California, Km 103 Carretera Tijuana - Ensenada. Ensenada, Baja California, \\ México. \\ *Correspondencia: excampos@gmail.com
}

\begin{abstract}
Resumen. Se presenta una lista de los isópodos de vida libre de la bahía de Todos los Santos con base en recolecciones alocrónicas realizadas entre 1988 y 2005 en el intermareal rocoso, arenoso y limo-arcilloso así como en el sublitoral arenoso hasta una profundidad de $256 \mathrm{~m}$. Se recolectaron 26 especies de las 48 registradas para la Provincia Californiana: 2 Asellota; 7 Cymothoida; 1 Oniscoidea; 7 Sphaeromatidea; 9 Valvifera Las especies más abundantes fueron Excirolana linguifrons (Richardson, 1899), (mesolitoral arenoso), Idotea fewkesi Richardson, 1905 junto con Idotea resecata Stimpson, 1857, (mesolitoral rocoso), Haliophasma geminatum Menzies et Barnard, 1959 (mesolitoral e infralitoral arcilloso asociada al pasto marino Zostera marina Linnaeus) y Caecognathia crenulatifrons (Monod, 1926), (sublitoral). Las especies recolectadas se ha registrado en biotopos marinos similares de California, Estados Unidos de América. De las 26 especies encontradas 7 son primeros registros para México: Erichsonella crenulata Menzies, 1950, Exosphaeroma amplicauda (Stimpson 1857), E. octonum (Richardson, 1899), Idarcturus allelomorphus Menzies et Barnard, 1959, Idotea fewkesi, Janiralata davisi Menzies, 1951 y Paracerceis gilliana (Richardson, 1899), extendiendo su distribución sureña hasta la bahía de Todos los Santos.
\end{abstract}

Palabras clave: Crustacea, Isopoda, vida-libre, Ensenada, Baja California, México.

\begin{abstract}
A checklist of the free-living isopods of Todos los Santos Bay, Baja California, is presented on the basis of allochronic collections made between 1988 and 2005 along the rocky, sandy, and muddy intertidal and sandy subtidal to 256 meters deep. We collected 26 of the 48 species known from the Californian province: 2 Asellota; 7 Cymothoida; 1 Oniscoidea; 7 Sphaeromatidea; 9 Valvifera. The most abundant species were Excirolana linguifrons (Richardson, 1899), (sandy mesolitoral), Idotea fewkesi Richardson, 1905 and I. resecata Stimpson 1857, (rocky intertidal), Haliophasma geminatum Menzies and Barnard, 1959, (muddy intertidal and subtidal associated with the eel grass Zostera marina), and Caecognathia crenulatifrons (Monod, 1926) (sublitoral). All the recorded species were found in similar marine biotopes in California, USA. Of the 26 species, 7 are recorded for the first time in Mexico, extending their southern distribution to Todos los Santos Bay. These are: Erichsonella crenulata Menzies, 1950, Exosphaeroma amplicauda (Stimpson 1857), E. octonum (Richardson, 1899), Idarcturus allelomorphus Menzies y Barnard, 1959, I. fewkesi, Janiralata davisi Menzies, 1951 and Paracerceis gilliana (Richardson, 1899).
\end{abstract}

Key words. Crustacea, Isopoda, free-living, Ensenada, Baja California, Mexico

\section{Introducción}

Los isópodos son uno de los grupos mejor conocidos de crustáceos bentónicos de la provincia Californiana. Trabajos clásicos sobre este componente faunístico incluyen los de Richardson (1899, 1905, 1909), Menzies (1950a, 1950b, 1951a, 1951b, 1952, 1954, 1957),

Recibido: 19 septiembre 2007; aceptado: 14 febrero 2008
Menzies y Barnard (1959) y Menzies y Miller (1972). Las contribuciones modernas para esta región biogeográfica incluyen principalmente las de Brusca (1983a, 1983b, 1984, 1989) y Brusca et al. (1995, 2001). Estos trabajos señalan que alrededor de 130 contribuciones sobre este grupo se han publicado para esta región biogeográfica, destacando los estudios sobre la costa del estado de California en los Estados Unidos de América (EUA). Las investigaciones sobre los isópodos de la costa de la 
península de Baja California son aún más escasos, razón por la cual muchas de las especies conocidas para esta provincia biogeográfica marcan su límite de distribución sureña en el área fronteriza de San Diego, California (véase, Menzies, 1962; Brusca y Wallerstein, 1977, 1979; Brusca, 1980, 1983a, b; Carvacho, 1981; Wägele, 1984; Delaney, 1984; Espinosa-Pérez y Hendrickx, 2006). El objetivo de este estudio es sintetizar el conocimiento sobre la diversidad de los isópodos de vida libre que residen en los diferentes biotopos de la bahía de Todos los Santos, colindante a Ensenada, Baja California, y documentar la información ecológica obtenida durante los trabajos de campo. En el Apéndice 1, se presenta la lista de las especies registradas.

\section{Material y método}

Área de estudio. La bahía de Todos los Santos se localiza a unos $100 \mathrm{~km}$ de la frontera México-EUA, sobre la costa oeste de Baja California, entre los $31^{\circ} 40^{\prime}$ y $31^{\circ} 56^{\prime} \mathrm{N}$ y los $116^{\circ} 36^{\prime}$ y $116^{\circ} 56^{\prime} \mathrm{O}$. Está delimitada al norte por punta San Miguel y al sur por punta Banda y las islas de Todos los Santos. Presenta una superficie de aproximadamente 25 000 ha con $18 \mathrm{~km}$ de largo y $14 \mathrm{~km}$ de ancho y el $90 \%$ tiene una profundidad entre los 10 y $50 \mathrm{~m}$. Presenta 2 entradas bien diferenciadas en su topografía submarina, divididas por 2 pequeñas islas situadas al oeste. La entrada noroeste, de $12 \mathrm{~km}$ de ancho, con profundidades menores a $50 \mathrm{~m}$, y la entrada suroeste, de $6 \mathrm{~km}$ de ancho, con profundidades hasta de $400 \mathrm{~m}$ en el cañón submarino formado entre punta Banda y las islas de Todos los Santos (Dirección General de Oceanografía y Señalamiento Marino, 1974). En 1a costa oriental de la bahía se encuentra 1ocalizado el estero de punta Banda, una laguna costera de $8 \mathrm{~km}$ de largo formada por un canal angosto, de poca profundidad (menor a $5 \mathrm{~m}$ ) en forma de "L" (Dirección General de Oceanografía y Señalamiento Marino, 1974). La variación anual de la temperatura superficial en la bahía de Todos los Santos oscila entre $\operatorname{los} 12^{\circ} \mathrm{C}$ (invierno) y $\operatorname{los} 22^{\circ} \mathrm{C}$ (verano). En general las mayores temperaturas dentro de la bahía se registran en la boca del estero de punta Banda y en el área aledaña al puerto de Ensenada, y las menores en la zona de punta San Miguel, donde no influye el efecto térmico de la bahía (Morales, 1977). En un plano general, la bahía de Todos los Santos puede considerarse como un área semiprotegida del efecto directo del oleaje, el que es disipado por las islas de Todos los Santos (Dirección General de Oceanografía y Señalamiento Marino, 1974). Walton (1955) clasificó a los sedimentos de la bahía en 3 tipos según su tamaño: 1) arena gruesa a media; 2) arena fina; 3) limo. A los que Riveroll (1985) agregó el de

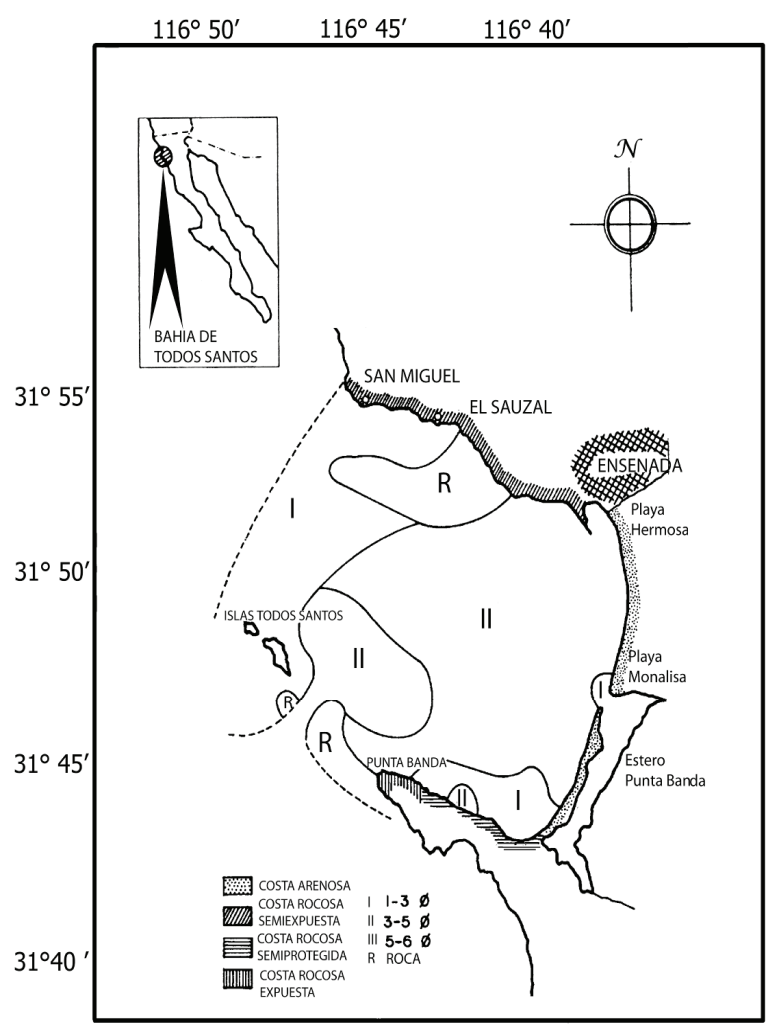

Figura 1. Carta de sedimentos en la bahía de Todos los Santos. I: arena gruesa a media; II: arena fina; III: limo R: Roca.

roca, la que está presente en una franja adyacente a la costa y en la zona entre mareas en la zona sur y centro de la bahía (Fig.1).

Las recolecciones y observaciones de campo fueron ejecutadas en tiempos diversos, desde marzo de 1988 hasta finales del 2005. Las especies intermareales se recolectaron en forma manual, inspeccionando macroalgas y tamizando sedimento en zonas rocosas y arenosas. Se realizó un total de 37 muestreos, visitando por lo general una sola vez 167 sitios que abarcaron las zona litoral a todo lo largo de la costa de la bahía y estero de punta Banda. Se ha incluido información proveniente de los cruceros "Bahía" I y II realizados en el B/O Francisco de Ulloa, del Centro de Investigación Científica y de Educación Superior de Ensenada(CICESE). Los cruceros se desarrollaron en junio y octubre de 1994. Se muestreó un total de 45 puntos hasta una profundidad de $256 \mathrm{~m}$ utilizando una draga McIntyre $\left(0.1 \mathrm{~m}^{2}\right)$. Las muestras se tamizaron con una malla de $0.5 \mathrm{~mm}$ de luz a bordo de la embarcación. Los ejemplares fueron preservados en formalina al $10 \%$, se identificaron en el laboratorio de zoología de invertebrados de la Facultad de Ciencias Marinas y quedaron depositados en la colección del bentos marino del CICESE. Con el objeto 
de comparar los resultados actuales con los publicados por Menzies (1962) en San Quintín, una bahía situada 350 km al sur del área de estudio, se realizó un análisis de afinidad por el método del índice de Jaccard (= IJS).

\section{Resultados y discusión}

Se recolectaron 26 de las 48 especies registradas para ambientes similares en el vecino estado de California, EUA. Todas se distribuyen en el Pacífico americano al sur de la provincia Californiana, formando parte de lo que Miller (1968) denominó "grupo del Pacífico sur". Sólo 4 fueron encontradas por Menzies (1962) en la bahía de San Quintín. Se considera que el bajo índice de similitud entre esta última bahía y la de Todos los Santos (ISJ=0.28) corresponde a la diferencia en hábitats, ya que en la de San Quintín el sustrato dominante es areno-fangoso. En el ambiente mesolitoral arenoso de la bahía de Todos los Santos la especie más abundante fue Excirolana linguifrons (Richardson, 1899). En la zona rocosa predominaron Idotea fewkesi Richardson, 1905 e Idotea resecata Stimpson, 1857. En la zona arcillosa y asociada con Zostera marina predominó Erichsonella crenulata Menzies, 1950. En el sublitoral de la bahía en ambiente de arena fina la especie más frecuente fue Caecognathia crenulatifrons (Monod, 1926). Schultz (1966) informó que esta especie, junto con Haliophasma geminatum Menzies et Barnard, 1959 es común en los cañones submarinos del sur del estado de California, EUA, encontrándose en 4 de 10 cañones muestreados. En arena fina sublitoral se localizó el 38\% de las especies recolectadas; en el intermareal rocoso el $23 \%$; entre algas el 27\%; en arena intermareal el $8 \%$ y entre vegetación sumergida el 4\%. (Cuadro 1). El $31 \%$ de las especies recolectadas se encontraron únicamente en la zona entre mareas. Sólo 2, Eurydice caudata Richardson, 1899 e Idarcturus allelomorphus Menzies et Barnard, 1959 (7\%), se encontraron en el sublitoral, en profundidades menores a $15 \mathrm{~m}$. El resto de las especies ocuparon amplios intervalos batimétricos dentro de las profundidades muestreadas. El mayor de éstos correspondió a $C$. crenulatifrons, entre los 16 y $256 \mathrm{~m}$. Por su parte Messanthura occidentalis Menzies et Barnard, 1959 fue registrada como especie rara, conocida únicamente por su holotipo (Wägele 1984), sin embargo, en la bahía de Todos los Santos 10 individuos fueron recolectados entre los 16 y $75 \mathrm{~m}$ en un sustrato de arena fina. De las especies recolectadas 7 nunca habían sido registradas de México; éstas son: Erichsonella crenulata Menzies, 1950, Exosphaeroma amplicauda (Stimpson, 1857), E. octonum (Richardson, 1899), I. allelomorphus, Idotea fewkesi, Janiralata davisi Menzies, 1951 y Paracerceis gilliana (Richardson, 1899).

\section{Agradecimientos}

A la Dra. Angelika Brandt, Universidad de Hamburgo, Alemania y al Dr. Michel Hendrickx, Instituto de Ciencias del Mar y Limnología de la UNAM, por el apoyo con literatura y a 2 revisores anónimos, por las sugerencias que mejoraron el artículo.

La autoría es alfabética.

\section{Literatura citada}

Austin, W. C. 1985. Isopoda. In An annotated checklist of marine invertebrates in the cold temperate northeast Pacific, British Columbia, Khoyatan Marine Laboratory (editions), 3:575587.

Bowman, T. E. 1977. Isopod crustaceans (except Anthuridae) collected on the Presidential Cruise of 1938. Proceedings of the Biological Society of Washington 89:653-666.

Brandt, A. y G. C. B. Poore. 2003. Higher classification of the flabelliferan and related Isopoda based on reappraisal of relationships. Invertebrate Systematics 17:893-923.

Brusca, R. C., y B. R. Wallerstein. 1977. The marine isopod Crustacea of the Gulf of California. I. Family Idoteidae. American Museum Novitates 2634: 1-17.

Brusca, R. C. y B. R. Wallerstein. 1979. Zoogeographic patterns of idoteid isopods in the northeast Pacific, with a review of shallow-water zoogeography for the region. Bulletin of the Biological Society Washington 3:67-105.

Brusca, R. C. 1980. Common intertidal invertebrates of the Gulf of California, segunda edición. University of Arizona Press, Tucson. 427 p.

Brusca, R. C. 1983a. Two new idoteid isopods from Baja California and the Gulf of California (Mexico) and an analysis of the evolutionary history of the genus Colidotea (Crustacea: Isopoda: Idoteidae). Transactions of the San Diego Society of Natural History 20:69-79.

Brusca, R. C. 1983b. A monograph on the isopod family Aegidae in the tropical Eastern Pacific. I. The genus Aegla. Allan Hancock Foundation, monographs in Marine Biology 12:1-39.

Brusca, R. C. 1984. Phylogeny, evolution and biogeography of the marine isopod subfamily Idoteinae (Crustacea: Isopoda: Idoteidae). Transactions of the San Diego Society of Natural History 20:99-134.

Brusca, R. C. 1989. Provisional keys to the genera Cirolana, Gnathia, and Limnoria known from California waters. SCAMIT Newsletter 8:17-21.

Brusca, R. C., R. Wetzer y S. France. 1995. Cirolanidae (Crustacea; Isopoda; Flabellifera) of the tropical eastern Pacific. Proceedings of the San Diego Society of Natural History 30:1-96

Brusca, R. C., V. Coelho y S. Taiti. 2001. A guide to the coastal isopods of California. Electronic publication: http://tolweb. org/notes/?note_id=3004 (consultado en febrero 2008).

Calderón, A. L. E. y J. F. Campoy. 1993. Bahía de Las Guásimas, estero Los Algodones y bahía de Lobos, Sonora. 
In Biodiversidad marina y costera de México, S. I. SalazarVallejo y N.E. González (eds.) CONABIO-CIQRO. México, D.F. p. 411-419.

Carvacho, A. 1981. Le genre Janira Leach, avec description d'une nouvelle espèce (Isopoda, Asellota). Crustaceana 41:131-142.

Carvacho, A. 1983. Asellota del golfo de California, con descripción de dos nuevos géneros y dos nuevas especies (Crustacea, Isopoda). Cahiers de Biologie Marine 24:281295.

Delaney, P. M. 1984. Isopods of the genus Excorallana Stebbing, 1904 from the Gulf of California, México (Crustacea, Isopoda, Corallanidae). Bulletin of Marine Science 31:1-20.

Dirección General de Oceanografía y Señalamiento Marítimo. 1974. Estudio geográfico de la región de Ensenada, Baja California. Secretaría de Marina, México, D.F. 463 p.

Espinosa-Pérez, M. C. y M. E. Hendrickx, 2001. Checklist of isopods (Crustacea: Peracarida: Isopoda) from the Eastern Tropical Pacific. Belgian Journal of Zoology 131:41-54.

Espinosa-Pérez, M. C. y M. E. Hendrickx, 2006. A comparative analysis of biodiversity and distribution of shallow-water marine isopods (Crustacea: Isopoda) from polar and temperate waters in the East Pacific. Belgian Journal of Zoology 136:219-247.

Glynn, P. W. y C. S. Glynn. 1974. On the systematics of Ancinus (Isopoda, Sphaeromatidae), with the description of a new species from the tropical eastern Pacific. Pacific Science 28:401-422.

Hatch, M. H. 1947. The Chelifera and Isopoda of Washington and adjacent regions. University of Washington Publications in Biology 10:155-274.

Iverson, E. W. 1974. Range extensions for some California marine isopod crustaceans. Southern California Academy of Sciences Bulletin 73:164-169.

Iverson, E. W. 1978. Status of Exosphaeroma inornata Dow and E. media George and Stromberg (Isopoda: Sphaeromatidae) with ecological notes. Journal of the Fishery Research Board of Canada 35:1381-1384.

Menzies, R. J. 1950a. The taxonomy, ecology, and distribution of northern California isopods of the genus Idotea with the description of a new species. Wasmann Journal of Biology 8:155-195.

Menzies, R. J. 1950b. Notes on California isopods of the genus Armadilloniscus, with the description of Armadilloniscus coronacapitalis n. sp. Proceedings of the. California Academy of Science 26:467-481.

Menzies, R. J. 1951a. New marine isopods, chiefly from northern California with notes on related forms. Proceedings of the United States National Museum 101:105-156.

Menzies, R. J. 1951b. A new species of Limnoria. Bulletin of the Southern California Academy of Science 50:86-88.

Menzies, R. J. 1952. Some marine asellote isopods from northern California, with descriptions of nine new species. Proceedings of the United States National Museum 102:117-159.

Menzies, R. J. 1954. A review of the systematics and ecology of the genus "Exosphaeroma" with the description of a new genus, a new species, and a new subspecies (Crustacea, Isopoda, Sphaeromidae). American Museum Novitates
1683:1-24

Menzies, R. J. 1957. The marine borer family Limnoriidae (Crustacea, Isopoda). Bulletin of Marine Science of the Gulf and Caribbean 7:101-200.

Menzies, R. J. y J. L. Barnard. 1959. Marine Isopoda on coastal shelf bottoms of Southern California: systematics and ecology. Pacific Naturalist 1:3-35.

Menzies, R. J. 1962. The marine isopod fauna of bahía de San Quintin, Baja California, Mexico. Pacific Naturalist 3:337348.

Menzies, R. J. y M. A. Miller 1972. Systematics and zoogeography of the genus Synidotea (Crustacea: Isopoda) with an account of Californian species. Smithsonian Contributions to Zoology 102:1-33.

Miller, M. A. 1968. Isopoda and Tanaidacea from buoys in coastal waters of the continental United States, Hawaii, and the Bahamas (Crustacea). Proceedings of the United States National Museum 125:1-53.

Morales, Z. C. 1977. Variaciones estacionales de la temperatura en la bahía de Todos los Santos, B. C. Ciencias Marinas 4:22-33.

Richardson, H. 1899. Key to the isopods of the Pacific coast of North America, with descriptions of twenty-two new species. Proceedings of the United States National Museum 21:815869.

Richardson, H. 1905. Monograph on the isopods of North America. Bulletin of the United States National Museum $54: 1-727$

Richardson, H. 1909. Isopods collected in the northwest Pacific by the U.S. Bureau of Fisheries Steamer "Albatross" in 1906. Proceedings of the United States National Museum 37:75129.

Riveroll, E. G. 1985. Distribución de materia orgánica en sedimentos de la bahía de Todos Santos, Baja California. Tesis, Facultad de Ciencias Marinas, Universidad Autónoma de Baja California, $26 \mathrm{p}$.

Schultz, G. A. 1964. Some marine isopod crustaceans from off the southern California coast. Pacific Science 18:307-314.

Shultz G. A. 1966. Marine Isopods of the submarine canyons of the southern Californian continental shelf. Allan Hancock Pacific Expeditions 27:1-56.

van der Heiden, M. A. y M. E. Hendrickx. 1982. Inventario de la fauna marina y costera del sur de Sinaloa, México. Instituto de Ciencias del Mar y Limnología, Estación Mazatlán, UNAM, Sinaloa. 135 p.

Vargas, A. J., H. K. Dean, D. Maurer y P. Orellana. 1985. Lista preliminar de invertebrados asociados a los sedimentos del golfo de Nicoya, Costa Rica. Brenesia 24:327-342.

Wägele J. W. 1984. Two new littoral Anthuridea from Baja California and redescription of Messanthura occidentalis (Crustacea: Isopoda). Zoologica Scripta 13:45-57.

Wallerstein, R. B. 1980. A taxonomic listing of common marine invertebrate species from southern California. In Isopoda. Technical Report of the Allan Hancock Foundation, D. Straughan y R.W. Klink (eds.). Washington, D.C. p. 230-236.

Walton W. R. 1955. Ecology of the living benthonic Foraminifera in Todos los Santos Bay, Baja California. Journal of Paleontology 29:952-1018. 
Wetzer, R., H. G. Kuck, P. Baéz, R. C. Brusca y L. M. Jurkevics 1991. Catalogue of the isopod Crustacea type collection of the Natural History Museum of Los Angeles County. Technical Report, Natural History Museum of Los Angeles County 3:1-59.

Wetzer R. y R. C. Brusca. 1997. Descriptions of the species of the suborders Anthuridea, Epicaridea, Flabellifera, Gnathoidea and Valvifera. In The Crustacea. Part 2: The Isopoda, Cumacea and Tanaidacea. Taxonomic atlas of the benthic fauna of the Santa Monica basin and Western Santa Barbara Channel, J. A. Blake y P. V. Scott(Eds.). Santa Barbara Museum of Natural History, California. p. 9-120.

Cuadro 1. Relación de las especies de isópodos registradas en bahía de Todos los Santos, Baja California, México, con el tipo de hábitat

\begin{tabular}{|c|c|c|c|c|c|c|}
\hline & Especies & $\begin{array}{l}\text { Sublitoral de } \\
\text { arena fina }\end{array}$ & $\begin{array}{l}\text { Roca } \\
\text { entremareas }\end{array}$ & $\begin{array}{l}\text { Arena } \\
\text { entremareas }\end{array}$ & $\begin{array}{l}\text { Sobre } \\
\text { algas }\end{array}$ & $\begin{array}{l}\text { Sobre } \\
\text { Zostera }\end{array}$ \\
\hline 1. & Messanthura occidentalis & $\mathrm{X}$ & & & & \\
\hline 2. & Joeropsis dubia dubia & $\mathrm{X}$ & & & & \\
\hline 3. & Heteroserolis carinata & $\mathrm{X}$ & & & & \\
\hline 4. & Exosphaeroma inornata & $\mathrm{X}$ & & & & \\
\hline 5. & Eurydice caudata & $\mathrm{X}$ & & & & \\
\hline 6. & Caecognathia crenulatifrons & $\mathrm{X}$ & & & & \\
\hline 7. & Caecognathia santaecrucis & $\mathrm{X}$ & & & & \\
\hline 8. & Idarcturus allelomorphus & $\mathrm{X}$ & & & & \\
\hline 9. & Edotia sublittoralis & $\mathrm{X}$ & & & & \\
\hline 10. & Synidotea magnifica & $\mathrm{X}$ & & & & \\
\hline 11. & Janiralata davisi & & $\mathrm{X}$ & & & \\
\hline 12. & Exosphaeroma amplicauda & & $\mathrm{X}$ & & & \\
\hline 13. & Exosphaeroma octonum & & $\mathrm{X}$ & & & \\
\hline 14. & Colidotea findleyi & & $\mathrm{X}$ & & & \\
\hline 15. & Ligia occidentalis & & $\mathrm{X}$ & & & \\
\hline 16. & Cirolana harfordi & & $\mathrm{X}$ & & & \\
\hline 17. & Ancinus granulatus & & & $\mathrm{X}$ & & \\
\hline 18. & Excirolana linguifrons & & & $\mathrm{X}$ & & \\
\hline 19. & Paracerceis gilliana & & & & $X$ & \\
\hline 20. & Paradella dianae & & & & $\mathrm{X}$ & \\
\hline 21. & Erichsonella crenulata & & & & $\mathrm{X}$ & \\
\hline 22. & Idotea aculeata & & & & $\mathrm{X}$ & \\
\hline 23. & Idotea fewkesi & & & & $\mathrm{X}$ & \\
\hline 24. & Idotea resecata & & & & $\mathrm{X}$ & \\
\hline 25. & Idotea stenops & & & & $\mathrm{X}$ & \\
\hline 26. & Haliophasma geminatum & & & & & $\mathrm{X}$ \\
\hline
\end{tabular}

Apéndice 1. Lista de especies registradas para la bahía de Todos los Santos (26). Los órdenes, familias y especies están ordenados alfabéticamente siguiendo la clasificación de Brandt y Poore (2003) y Espinosa Pérez y Hendrickx (2006).

\section{ASELLOTA Latreille, 1802}

JANIRIDAE Sars, 1897

1. Janiralata davisi Menzies, 1951.

Material examinado: 1 ejemplar, entre algas en una poza de la zona rocosa intermareal, bahía de Todos los Santos, Baja California, México (bTS, BCMéx) 
Nueva distribución. Desde Monterrey, California, Estados Unidos de América (EUA) hasta bTS, BCMéx

(Menzies, 1951a; este estudio).

JOEROPSIDAE Nordenstam, 1933

2. Joeropsis dubia dubia (Menzies, 1951).

Material examinado: 1 ejemplar en el sublitoral a una profundidad 75 m, bTS, BCMéx.

Distribución. Desde Newport Bay, California, EUA hasta bahía de San Quintín, costa oeste de Baja California y desde laguna Percebú a bahía Concepción, golfo de California, México (Menzies, 1962; Carvacho, 1983; Wetzer et al., 1991).

CYMOTHOIDA Wägele, 1989

\section{ANTHURIDAE Leach, 1814}

3. Haliophasma geminatum Menzies and Barnard, 1959.

Material examinado: 13 ejemplares en la zona sublitoral del estero de punta Banda (profundidad $1 \mathrm{~m}$ ) asociados a Zostera marina, bTS, BCMéx.

Distribución. Desde Puget Sound, Washington, EUA hasta bahía de San Quintín, costa oeste de Baja California,

México, (Menzies y Barnard, 1959; Menzies, 1962; Schultz, 1964; Austin, 1985).

4. Messanthura occidentalis Menzies y Barnard, 1959.

Material examinado: 10 ejemplares en arena fina entre 25 y 63 metros de profundidad, bTS, BCMéx.

Distribución. Desde punta Concepción California, EUA hasta el golfo de Nicoya, Costa Rica, incluyendo el golfo de California (Brusca et al., 2001).

CIROLANIDAE Dana, 1852

5. Cirolana harfordi (Lockington, 1877).

Material examinado: 16 ejemplares en la zona rocosa entremareas, asociados a los tubos del anélido colonial Phragmatopoma californica, bTS, BCMéx.

Distribución. Desde la isla de Vancouver, Canadá hasta bahía Magdalena, costa oeste de Baja California Sur, México; un registro dentro del golfo de California en La Paz, Baja California Sur, México (Brusca et al., 1995).

6. Eurydice caudata Richardson, 1899.

Material examinado: 5 ejemplares en el sublitoral entre 7 y 43 metros de profundidad, bTS, BCMéx.

Distribución. Desde San Diego, California, EUA hasta La Libertad, Ecuador, incluyendo el golfo de California,

México. Islas Guadalupe, Revillagigedo, Coco y Galápagos (Bowman, 1977; Brusca et al., 1995; Wallesrstein, 1980).

7. Excirolana linguifrons (Richardson, 1899).

Material examinado: 12 ejemplares en zona arenosa entremareas, bTS, BCMéx.

Nueva distribución. Desde Monterey, California, EUA hasta bTS, BCMéx (Richardson, 1905; Brusca et al., 2001, este estudio).

GNATHIIDAE Leach, 1814

8. Caecognathia crenulatifrons (Monod, 1926).

Material examinado: 18 ejemplares entre 14 y 136 metros de profundidad en el sublitoral, bTS, BCMéx.

Nueva distribución. Desde Point Santa Cruz, California, EUA hasta bTS, BCMéx (Iverson, 1974; Brusca et al., 2001; este estudio).

9. Caecognathia santaecrucis (Schultz, 1972)

Material examinado: 1 ejemplar en el sublitoral a los 76 metros de profundidad, bTS, BCMéx.

Nueva distribución. Desde el sur de California, EUA hasta bTS, BCMéx (Brusca et al., 2001; este estudio).

\section{ONISCOIDEA Latreille, 1802}

\section{LIGIIDAE Brandt, 1883}

10. Ligia occidentalis (Dana, 1853)

Material examinado: 20 ejemplares en la zona rocosa alta intermareal, bTS, BCMéx.

Distribución. Desde Oregon, EUA hasta bahía Chamela, Jalisco, incluyendo el golfo de California, México

(Richardson, 1905; Austin, 1985; Bowman, 1977; Espinosa-Pérez y Hendrickx, 2001).

ANCINIDAE Dana, 1852

11. Ancinus granulatus Holmes y Gay, 1909 
Material examinado: 4 ejemplares en arena en el estero de punta Banda, bTS, BCMéx.

Distribución. Desde el sur de California, EUA, hasta isla Cedros, en la costa oeste de Baja California, México y desde San Felipe (golfo de California), Baja California hasta Mazatlán, Sinaloa, México (Glynn y Glynn, 1974; Wallerstein, 1980; van der Heiden y Hendrickx, 1982).

SPHAEROMATIDAE Latreille, 1825

12. Exosphaeroma amplicauda (Stimpson 1857)

Material examinado: 2 ejemplares en una poza entremareas en la zona litoral rocosa, bTS, BCMéx.

Distribución. Desde Kyska Harbor, Alaska, EUA hasta bTS, BCMéx (Richardson,1905; Hatch 1947; Brusca et al., 2001; este estudio).

13. Exosphaeroma inornata Dow, 1958

Material examinado: 1 ejemplar en la zona sublitoral a 25 metros de profundidad, bTS, BCMéx.

Distribución. Desde Puget Sound, Washington, EUA hasta bTS, BCMéx (Iverson, 1978; Brusca et al., 2001; este estudio).

14. Exosphaeroma octonum (Richardson, 1899)

Material examinado: 1 ejemplar en una poza entremareas en la zona litoral rocosa, bTS, BCMéx.

Distribución. Desde Tomales Bay, California, EUA hasta bTS, BCMéx (Iverson, 1978; Brusca et al., 2001; este estudio).

15. Paracerceis gilliana (Richardson, 1899)

Material examinado: 3 ejemplares entre algas en la zona rocosa inferior, bTS, BCMéx.

Nueva distribución. Desde Mendocino county, California, EUA hasta bTS, BCMéx (Richardson, 1905; Brusca et al., 2001; este estudio).

16. Paradella dianae (Menzies, 1962)

Material examinado: 1 ejemplar entre algas en la zona rocosa, bTS, BCMéx.

Distribución. Desde Los Ángeles, California, EUA, hasta San Juan de Alima, Michoacán, México. Incluyendo el golfo de California, Indo Pacífico, Atlántico y Mediterráneo. (Menzies, 1962; Iverson, 1974; Espinosa-Pérez y Hendrickx, 2001).

SEROLIDAE Dana, 1852

17. Heteroserolis carinata (Lockington 1857).

Material examinado: 5 ejemplares en el sublitoral entre 19 y 29 metros de profundidad, bTS, BCMéx.

Distribución. Desde Santa Mónica Bay, California, EUA, hasta bahía de San Quintín, costa oeste de Baja California, México; desde isla Ángel de la Guarda, Baja California hasta cabo San Miguel y de isla Tiburón hasta bahía Lobos, Sonora, en el golfo de California, México (Menzies y Barnard, 1959; Calderón y Campoy, 1993; Espinosa-Pérez y Hendrickx, 2001; Wetzer y Brusca, 1997).

VALVIFERA Sars, 1882

ARCTURIDAE Dana, 1849

18. Idarcturus allelomorphus Menzies y Barnard, 1959.

Material examinado: 2 ejemplares en el sublitoral entre los 7 y 34 metros de profundidad, bTS, BCMéx.

Distribución. Desde Monterey, California, EUA hasta bTS, BCMéx (Menzies y Barnard, 1959; Iverson, 1974; Brusca et al., 2001).

IDOTEIDAE Samouelle, 1819

19. Colidotea findleyi Brusca y Wallerstein 1979.

Material examinado: 2 ejemplares entre algas en la zona rocosa entremareas, bTS, BCMéx.

Distribución. Desde San Diego, California, EUA hasta (sic.) punta San Eugenio, sobre la costa oeste de Baja California (probablemente punta Eugenia, Baja California Sur, México); San Felipe, Baja California, Puerto Peñasco a Punta Lobos, Sonora en el golfo de California; Isla Guadalupe, México (Westzer et al., 1991; Brusca et al., 2001).

20. Edotia sublittoralis Menzies y Barnard, 1959.

Material examinado: 14 ejemplares en el sublitoral entre 14 y 43 metros de profundidad, bTS, BCMéx.

Distribución. Desde Vancouver Island, Canadá hasta Newport, California, EUA y bTS, BCMéx. Un registro en el golfo de Nicoya, Costa Rica (Vargas et al., 1985; Brusca et al, 2001).

21. Erichsonella crenulata Menzies, 1950.

Material examinado: 25 ejemplares en el estero de punta Banda asociados al pasto marino Zostera marina, bTS, BCMéx.

Distribución. Desde el sur de California, EUA hasta bahía de San Quintín, costa oeste de Baja California, México (Menzies, 1950a; 1962).

22. Idotea aculeata (Stafford, 1913 ). 
Material examinado: 2 ejemplares asociados a algas en la zona rocosa entremareas, bTS, BCMéx.

Distribución. Desde Columbia Británica, Canadá, hasta isla Cedros, sobre la costa oeste de Baja California, México; desde Guaymas, Sonora hasta La Paz, en el golfo de California, México (Menzies, 1950a; Brusca y Wallestein, 1977; Austin, 1985; Calderón y Campoy, 1993; Brusca et al., 2001).

23. Idotea fewkesi Richardson, 1905.

Material examinado: 2 ejemplares asociados a Macrocystis pyrifera en la zona sublitoral a 5 metros de profundidad, bTS, BCMéx.

Nueva distribución. Desde el golfo de Alaska, EUA hasta bTS, BCMéx, (Hatch, 1947; Brusca et al., 2001; este estudio).

24. Idotea resecata Stimpson 1857.

Material examinado: 1 ejemplar asociado a Macrocystis pyrifera arrojada a la playa, bTS, BCMéx.

Distribución. Desde Karta Bay, golfo de Alaska, EUA, hasta (sic.) bahía Tórtola, costa oeste de Baja California, (probablemente bahía Tortugas, Baja California Sur), México; la Paz, Cabo San Lucas y Rocas Alijos en el golfo de California, México (Brusca y Wallerstein, 1977; Austin, 1985; Brusca et al., 2001).

25. Idotea stenops Benedict, 1898.

Material examinado: 3 ejemplares asociados a Macrocystis pyrifera en la zona sublitoral a 5 metros de profundidad, bTS, BCMéx.

Distribución. Desde Alaska, USA, hasta (sic.). Punta San Eugenio (probablemente punta Eugenia, Baja California Sur), sobre la costa oeste de Baja California, México. Desde punta San Telmo hasta La Paz, Baja California Sur en el golfo de California (Brusca y Wallerstein, 1977; Austin, 1985; Brusca et al., 2001).

26. Synidotea magnifica Menzies y Barnard, 1959.

Material examinado: 4 ejemplares en el sublitoral entre 24 y 51 metros de profundidad, bTS, BCMéx.

Nueva distribución. Desde San Luis Obispo, California, EUA hasta bTS, BCMéx (Menzies y Barnard, 1959; Brusca et al., 2001, este estudio). 